\title{
Management der digitalen Transformation
}

\author{
Ein Werkstattbericht aus der Technischen Universität Kaiserslautern
}

\section{Wie das Thema Management der digitalen Transformation erfolgreich in die zentralen Tätigkeitsfelder einer jeden Professur - Forschung, Lehre und Praxis - integriert werden kann, steht im Mittelpunkt dieses Beitrags. Hierfür wird ein Werkstattbericht von dem Fachgebiet für Strategie, Innova- tion und Kooperation der Technischen Universität Kaiserslautern geliefert.}

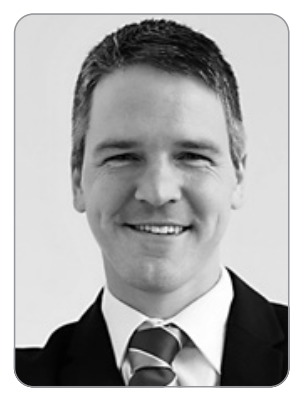

Prof. Dr. Gordon Müller-Seitz ist Leiter des Fachgebiets für Strategie, Innovation und Kooperation an der Technischen Universität Kaiserslautern. Bevorzugte Forschungsgebiete: Strategie, Innnovations- und Technologiemanagement, Digitalisierung, Kooperation und Koordination, Netzwerke, Risiko und Krisen.

Summary: How to address managing the digital transformation against the backdrop of the key areas of activity of a professorship - research, teaching and praxis - is of key interest in this article. Towards this end, a pragmatic work-in-progress report from the Technische Universität Kaiserslautern's Chair of Strategy, Innovation and Cooperation is offered.

Stichwörter: Management der digitalen Transformation, Strategie, Innovation, Kooperation, Lehre, Forschung, Drittmittelpraxis

\section{Problemstellung: Relevanz der Digitalisierung erkannt, Umsetzung gestartet}

"In the digital age of 'overnight' success stories such as Facebook, the hard slog is easily overlooked." (vgl. Dyson zitiert nach Kor, 2019)

Für die Technische Universität Kaiserslautern (TUK) stellt die Digitalisierung laut des Hochschulentwicklungsplans 2025 „ein zentrales Querschnittsthema [...] mit strategischer Bedeutung für die Bereiche Forschung, Lehre, Transfer und Administration“ (vgl. Technische Universität Kaiserslautern, 2019: 30) dar. Auch der Fachbereich Wirtschaftswissenschaften der TUK, an dem der Autor die- ses Beitrags das Fachgebiet Strategie, Innovation und Kooperation leitet, stuft die Relevanz des Themas hoch ein, weshalb alle beteiligten Akteure an einem Strang ziehen.

Angesichts dieser gemeinhin anerkannten Relevanz stellt sich nunmehr für jede Professur letztlich die Frage, welche und wie Themen des Managements der digitalen Transformation in Forschung, Lehre und Praxis konkret adressiert werden können. Im Folgenden wird anhand des Fallbeispiels des eigenen Fachgebiets vorgestellt, mit Hilfe welcher Maßnahmen das Management der digitalen Transformation in die zentralen Handlungsfelder Forschung, Lehre und Praxis integriert wurde. Der Beitrag hat dabei insofern einen dezidierten ,Werkstatt'-Charakter, als das breite Spektrum an Aktivitäten des Fachgebiets keineswegs ausschließlich auf starren oder vermeintlich perfekt ausgearbeiteten Maßnahmen basiert. Vielmehr haben die Aktivitäten oftmals experimentellen Charakter und sind durchaus arbeitsintensiv, da quasi per ,Trial-and-Error' immer wieder Neues ausprobiert wird, weshalb hier Parallelen zu dem voranstehenden Zitat von Dyson existieren.

\section{Aktivitäten in Forschung, Lehre und Praxis}

Der Autor ist in Forschung, Lehre und Praxis schon seit langer Zeit mit der Digitalisierung thematisch befasst. Plakativ ließe sich formulieren, dass das Management der digitalen Transformation zur DNA des Fachgebiets gehört.

Nachstehend sollen nun skizzenhaft Einblicke in Forschung (2.1), Lehre (2.2) und Praxisaktivitäten (2.3) mit Bezug zum Management der digitalen Transformation gegeben werden. Diese isolierte Betrachtung wird dann im nächsten Schritt in Kapitel 3 zugunsten einer Betrachtung der Schnittstellen zwischen den Feldern aufgehoben (3; s. voranstehende Darstellung). 
Quelle: Eigene Darstellung.

Darst. 1: Übersicht über die miteinander eng verzahnten Werkstattaktivitäten zum Management der digitalen Transformation am Fachgebiet

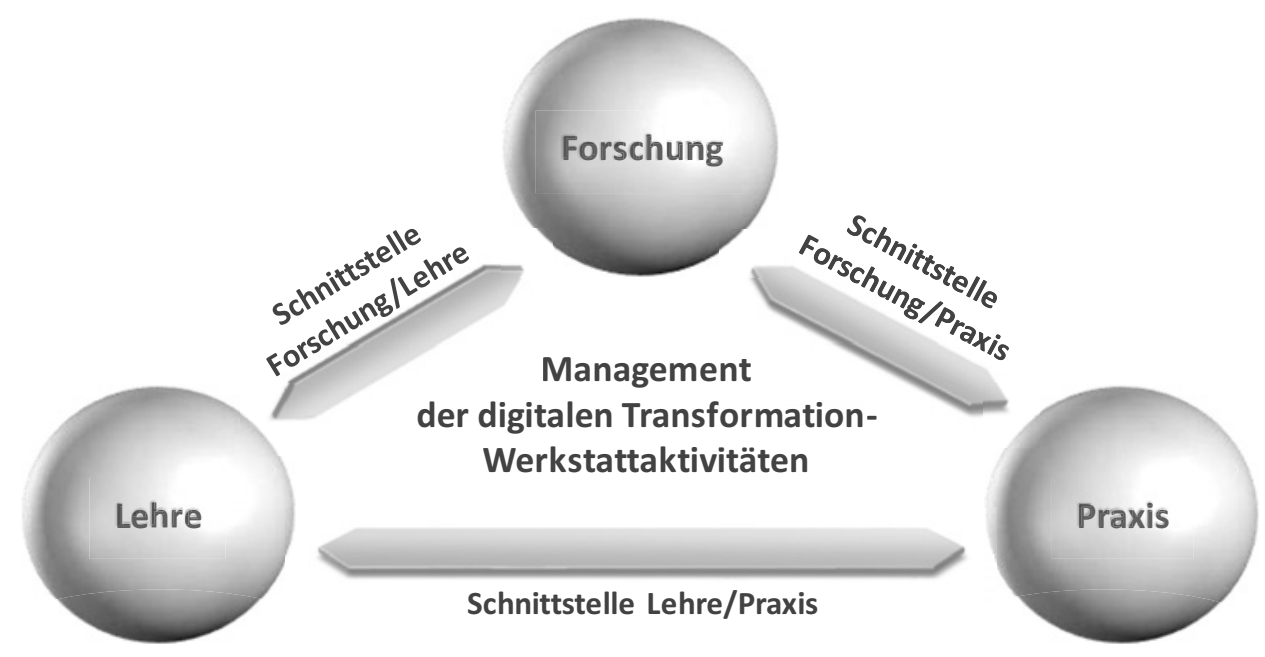

\subsection{Forschung}

Das Management der digitalen Transformation wird durch den Autor seit seiner Promotion erforscht (vgl. exemplarisch Kaiser/Müller-Seitz/Lopes/Cunha, 2007) und wurde aus den Perspektiven der Strategie-, Innovations- und Netzwerkforschung sowie des Personalmanagements beleuchtet. Beispiele sind Studien zu den betriebswirtschaftlichen Implikationen von Open Source Software, zu technologischen Pfadabhängigkeiten, innovativen Formen der Vernetzung oder agilen Managementmethoden (s. exemplarisch Dobusch/Dobusch/Müller-Seitz, 2019; Hilgers/ Müller-Seitz/Piller, 2010; Lange/Sydow/Müller-Seitz/Windeler, 2013).

Die Forschungsaktivitäten beschränken sich jedoch nicht nur isoliert auf das Fachgebiet, sondern umfassen mittlerweile ein breites Spektrum an Kooperationen innerhalb und außerhalb des Fachbereichs. Zuvorderst sei auf die fachbereichsinterne Kooperation mit Kolleginnen und Kollegen aus den Bereichen Controlling, Entrepreneurship, Management Studies, Marketing und Personalmanagement verwiesen. Seit mittlerweile drei Jahren erfolgt eine Verzahnung von Forschungsaktivitäten mit der Zielsetzung, die digitale Transformation aus dezidiert managementorientierter Perspektive zu untersuchen. Illustrativ sei in diesem Zusammenhang auf den programmatisch ausgerichteten Sammelband zum Management der digitalen Transformation verwiesen (vgl. Lingnau/Müller-Seitz/Roth, 2018). Dort sind die unterschiedlichen Perspektiven der Fachgebiete sowie das Potential für interdisziplinäre Kooperationen dargelegt.

Dies ist insofern von Belang, als der wissenschaftliche Diskurs hierzu in der Betriebswirtschaftslehre noch vergleichsweise gering ausgeprägt ist. Disziplinen wie etwa die Ingenieurswissenschaften oder Informatik können in diesem Zusammenhang schon auf eine längere Tradition zurückblicken. Aufgrund des technischen Charakters und der Stärke der ingenieurswissenschaftlichen beziehungsweise informatikgetriebenen Fachbereiche an der TUK bietet es sich an, auch hier Kooperationen einzugehen. Denn so kann letztlich aus Sicht des Autors in der Managementforschung verankertes, aber zugleich interdisziplinär orientierte innovative Grundlagenforschung erfolgreich vorangetrieben werden. Als ein Beispiel sei hier die Kooperation mit dem Deutschen Forschungszentrum für Künstliche Intelligenz sowie der Smart Factory ${ }^{\mathrm{KL}}$, der weltweit ersten herstellerunabhängigen Demonstrations- und Forschungsplattform zur Umsetzung einer intelligenten Fabrik, und der damit verbundenen Professur für Produktionsautomatisierung angeführt (vgl. Müller-Seitz/Zühlke/Braun/Gorecky/Thielen, 2018). Anhand der Smart Factory ${ }^{\mathrm{KL}}$ wurde analysiert, inwieweit netzwerkbasierte Geschäftsmodellinnovationen vorangetrieben werden können (vgl. Thielen, 2019). Ein aktuelles universitätsübergreifendes Beispiel ist das Projekt des Jubiläumsfonds der österreichischen Nationalbank zu ,Digital Transformation as Path Development of Hidden Champions'. In diesem Projekt unter der Leitung des Kollegen Güttel (Johannes Kepler Universität Linz) wird in einem Forschungskollektiv der Frage nachgegangen, wie mittelständische Hidden Champions mit den Herausforderungen der digitalen Transformation umgehen. Nebst dem Ziel, empirische Studien durchzuführen, dient das Projekt auch dem informellen Wissenstransfer zwischen Professuren von Universitäten in Österreich, Deutschland, Liechtenstein und Norwegen sowie den am Projekt beteiligten mittelständischen Unternehmen.

Schlussendlich sei noch auf europaweit ausgerichtete Forschungsambitionen verwiesen. Hier besteht eine Kooperation mit dem Joint Research Council (JRC) der Europäischen Union. Das JRC ist ein Think Tank, der u. a. transdisziplinäre Workshops organisiert sowie Forschungsprojekte am Center for Advanced Studies ermöglicht. Im Herbst 2019 wurde beispielsweise ein Workshop ,Digital Transformation' ausgerichtet. Die Gäste des Workshops 
wurden gezielt aufgrund ihrer verschiedenen Hintergrunde eingeladen, um so unterschiedliche Sphären aufeinanderprallen $\mathrm{zu}$ lassen und über innovative Lösungsansätze für aktuelle und künftige Herausforderungen der digitalen Transformation zu diskutieren. Die Teilnehmenden aus der Wissenschaft rekrutierten sich aus den Bereichen der Volkswirtschaftslehre, Psychologie, Soziologie, Informatik sowie Politikwissenschaften und wurden durch Vertreter aus Politik, Industrie und Kunst ergänzt. Dieses lose Kooperationsangebot wird kontinuierlich - im besten Sinne des hier angedachten Werkstattberichts - weiterentwickelt und erweist sich nicht nur als hervorragende inhaltliche Bereicherung, sondern führt auch zur Erweiterung des Netzwerks des Fachgebiets.

\subsection{Lehre}

- Mit Blick auf die Lehre wird das Thema der Digitalisierung in Form von zwei Aspekten adressiert: zum einen inhaltlich durch die Integration digitalisierungsrelevanter Themen in das Curriculum, zum anderen durch die Digitalisierung der Lehre. Hinsichtlich des Curriculums findet sich das Management der digitalen Transformation vor allem in der fachbereichsinternen Kooperation sowie in der generellen Kooperation in interdisziplinären Studiengängen zu Wirtschaftsinformatik und -ingenieurswesen wieder. Hier wurde ein gemeinsamer Masterschwerpunkt ,Management der digitalen Transformation' mit den Professuren für Entrepreneurship, Management Studies, Marketing und Personalmanagement ins Leben gerufen. Ein so genanntes Integrationsmodul ist dabei obligatorischer Bestandteil des Schwerpunkts. Dort wird das Thema Geschäftsmodellinnovationen aus den Perspektiven verschiedener Professuren in der ersten Vorlesungshälfte des Semesters zunächst theoretisch-konzeptionell beleuchtet. Zwischenpräsentationen zum theoretisch-konzeptionellen Teil bilden sodann den Aufschlag für eine Kooperation mit der Unternehmungspraxis. Die zweite Vorlesungshälfte ist durch die Bearbeitung einer realen Herausforderung des Kooperationspartners aus der Praxis gekennzeichnet und endet auch mit einer Abschlusspräsentation - üblicherweise in den Räumlichkeiten des Praxispartners, um so für ein für die Berufspraxis realistisches Flair zu sorgen. Weitere Vertiefungsmöglichkeiten mit Bezug zur digitalen Transformation bestehen und werden jeweils von zwei der zuvor genannten Professuren angeboten.

- Unabhängig von der fachbereichsinternen Kooperation werden Fragestellungen zur Digitalisierung auch im Curriculum des Fachgebiets aufgegriffen. In erster Linie geschieht dies in den Masterveranstaltungen,Strategie und Technologie', aber auch ,Innovation Management' und
,Managing interorganizational Relations'; allesamt Veranstaltungen, denen auch Monographien aus eigener Feder zugrunde liegen (vgl. Corsten/Gössinger, Müller-Seitz, 2017; Lingnau/Müller-Seitz/Roth， 2018; Müller-Seitz/ Weiss, 2019; Sydow/Schüßler/Müller-Seitz, 2016). Schließlich wird in digital gestützter Form auch das an der TUK angesiedelte Distance and Independent Studies Center (DISC) - einer der in Deutschland erfolgreichsten Anbieter postgradualer Fernstudiengänge - mit Inhalten zum Management der digitalen Transformation durch das Fachgebiet unterstützt.

- Die Aktivitäten am DISC stellen sodann auch die Brücke zur Nutzung der Digitalisierung der Lehre dar. Dies ermöglicht es dem Fachgebietsteam, die Lehre teilweise in Form des Flipped-classroom-Konzepts anzubieten. Hier werden die grundlegenden Vorlesungsinhalte im Intranetportal eine Woche vor der jeweiligen Veranstaltung online bereitgestellt. Im Rahmen der eigentlichen Veranstaltung werden dann aktuelle Fallbeispiele aus der Organisationspraxis aufgegriffen und vor dem theoretisch-konzeptionellen Hintergrund der jeweiligen Videoeinheit gemeinsam im Plenum erörtert. Dieses Konzept kommt sowohl im Rahmen von Bachelor- als auch Masterveranstaltungen zum Einsatz und erweist sich als sehr populär bei den Studierenden und den Lehrenden. So ist eine effektive Fortschrittskontrolle in Form von Fragen und Diskussionsrunden in den Vorlesungseinheiten möglich, während die Grundlagen digital zeit- und ortsunabhängig abgerufen werden können.

\subsection{Praxis}

- Der Austausch mit der Praxis wird gezielt gesucht, um so Handlungsspielräume zu erweitern und hinsichtlich der Herausforderungen und Bedarfe der Unternehmenspraxis am Puls der Zeit zu sein. Überdies erhöht es die Employability der Mitarbeiterinnen und Mitarbeiter des Fachgebiets sowie der Studierenden.

- Als Herzstück der interdisziplinären Kooperation mit der Unternehmungspraxis sei auf das durch das Bundesministerium für Wirtschaft und Energie geförderte Mittelstand 4.0 Kompetenzzentrum Kaiserslautern verwiesen. $\mathrm{Zu}$ den Konsortialpartnern zählen neben dem Fachgebiet für Strategie, Innovation und Kooperation sowie Praxispartnern des Rheinland-pfälzischen Mittelstands auch noch die Smart Factory ${ }^{\mathrm{KL}}$, das Deutsche Forschungszentrum für künstliche Intelligenz, das Fachgebiet für Virtuelle Produktentwicklung der TUK sowie das Institut für Technologie und Arbeit. Zielsetzung des Zentrums ist es, kleine und mittelständische Unternehmen (KMU) in Digitalisierungsfragen zu unterstützen. Das Fachgebiet übernimmt dabei die Leitung für Fragestellungen, die aus strategischen Management sowie dem Innovations- und 
Netzwerkmanagement stammen, speziell hinsichtlich der Entwicklung neuer Geschäftsmodelle.

- So ermöglicht ein Online-Readiness-Check zur Digitalisierungsfähigkeit es bisher über 1.300 Unternehmen, den eigenen Digitalisierungsgrad $\mathrm{zu}$ bestimmen und branchenübergreifend zu vergleichen. Ideenwerkstätten bauen auf diesem Readiness-Check auf und dienen der Identifikation von Digitalisierungspotentialen sowie der Ableitung einer Digitalisierungsstrategie. Die maßgeschneidert zusammengestellten, interdisziplinären Teams unterstützen bei der Implementierung neuester Technologien und Digitalisierungslösungen.

- Eingebettet werden die durch das Fachgebiet unterstützten Praxisformate in das erweiterte Wertschöpfungsnetzwerk WERNER, welches gleichzeitig als branchenübergreifende Online-Plattform zur Vernetzung von Unternehmen dient. Das wachsende Netzwerk bietet Unternehmen die Möglichkeit, potenzielle Technologiepartner zu identifizieren und Kooperationsprojekte zu initiieren. Die nachhaltige Auslegung dieses Angebots begünstigt eine langfristige Zusammenarbeit und bietet eine Übersicht bereits erfolgreich umgesetzter Digitalisierungsprojekte.

- Als eines der ersten der mittlerweile bundesweit 26 Kompetenzzentren koordiniert das Kompetenzzentrum in Kaiserslautern darüber hinaus Trainer im Bereich Künstlicher Intelligenz, um KMU sowie dem Handwerk spezielle Unterstützung zur Anwendung Künstlicher Intelligenz zu bieten. Dadurch wird der Transfer von wettbewerbskritischem Wissen im Feld der Künstlichen Intelligenz in den Unternehmen und die Anwendung vor Ort vorangetrieben. Relevante Anwendungsfelder sind u. a. Assistenzsysteme, Smart-Data-Analysen und Intelligente Produkte und Services.

- Durch das breite Leistungsportfolio und sein ausgeprägtes interorganisationales Netzwerk etabliert das Mittelstand 4.0 Kompetenzzentrum Kaiserslautern langfristige Strukturen für das Management der digitalen Transformation. Die interdisziplinären Konsortialpartner bringen im Rahmen dieser Formate Inhalte aus der Theorie immer wieder in die Unternehmenspraxis ein und sammeln wiederum wichtige Erkenntnisse für die Forschung. Im Rahmen der Lehre haben auch Studierende am Mittelstand 4.0 Kompetenzzentrum Kaiserslautern die Möglichkeit, durch Praxisarbeiten Einblicke in die Wirtschaft zu erlangen.

\section{Nutzung von Synergien zwischen Forschung, Lehre und Praxis}

Wie aus den voranstehenden Ausführungen bereits ersichtlich wurde, kommt es naturgemäß immer wieder zu Überschneidungen zwischen den drei beschriebenen Sphären. Die zuvor ,in Reinkultur' präsentierten Säulen Forschung, Lehre und Praxis sollen daher auf konkrete Schnittstellen hin untersucht werden, um aufzuzeigen, wie sich Synergien zwischen Forschung und Lehre (3.1), Forschung und Praxis (3.2) und Lehre und Praxis (3.3) generieren lassen.

\subsection{Schnittstelle Forschung/Lehre}

Neben der Nutzung von aktuellen Fallbeispielen kommt es kontinuierlich zur Integration eigener Forschungsarbeiten in die Lehre, unabhängig davon, ob es sich um Veranstaltungen im Bachelor- oder Masterbereich handelt. Hierdurch ist es möglich, die Studierenden bereits frühzeitig für eigene empirische Erhebungen $\mathrm{zu}$ sensibilisieren und für aktuelle Fragestellungen zum Management der digitalen Transformation zu interessieren. Dabei sollen die unterschiedlichen Theorien und Bezugsrahmen nicht nur auf die betreffenden Phänomene der Digitalisierung angewandt werden. Vielmehr soll auch eine kritische Betrachtung der Limitationen der jeweiligen Ansätze erfolgen, um so zu einem reflektierten Umgang mit Wissenschaft und Praxis anzuregen. Hierdurch werden die Studierenden en passant auch an das wissenschaftliche Arbeiten an sich herangeführt (vgl. Müller-Seitz/Braun, 2013).

Im Einklang mit den Forschungsaktivitäten des Fachgebiets wird in erster Linie auf qualitative Methoden der Sozialforschung Wert gelegt. Die entsprechenden Inhalte werden im Rahmen von Übungen sowie im Zuge der Abschlussarbeiten im Bachelor- und Masterbereich vermittelt. Im Anschluss an methodische Grundlegungen (vgl. DannerSchröder/Müller-Seitz, 2017) wird im Rahmen von Datensammlung und -analyse dezidiert auf die Digitalisierung und die damit verbundenen Möglichkeiten und Grenzen aus methodischer Sicht näher eingegangen.

Als Beispiel sei in diesem Zusammenhang auf die Analyse von Facebook-Gruppen zur Bewältigung der Flüchtlingskrise (2015-2018) im Rahmen der fachbereichsinternen Kooperation zum Management der digitalen Transformation verwiesen (vgl. Danner-Schröder/Müller-Seitz, im Druck). Dabei wurden die Studierenden angehalten, ChatVerläufe verschiedener Facebook-Gruppen als Form der Datensammlung abzuspeichern und die Daten systematisch aufzubereiten. So erhielten die Studierenden nicht nur das damit verbundene qualitativ-explorative Rüstzeug für die Datenanalyse mit Blick auf Bildung, Gewichtung und Kategorisierung von sich emergent ergebenden Themen. Vielmehr galt es auch komplexe inhaltliche Zusammenhänge aufzuspüren, etwa wie Kooperation und Koordination innerhalb verschiedener Facebook-Gruppen erfolgte.

\subsection{Schnittstelle Forschung/Praxis}

Die Forschungsaktivitäten des Fachgebiets verfolgen das Ziel, praxisrelevante Phänomene des Managements der 
digitalen Transformation interdisziplinär, empirisch und zugleich theoretisch fundiert zu untersuchen. Die dadurch entstehenden Erkenntnisse sind daher nicht nur für Fachleute aus dem Bereich der Forschung von Interesse, sondern setzen auch Impulse für die Unternehmungspraxis.

Insofern wird nicht nur darauf Wert gelegt, bei hochrangigen Fachpublikationen zu reüssieren. Vielmehr werden bewusst auch Transferpublikationen ins Visier genommen (vgl. exemplarisch Müller-Seitz/Beham/Thielen, 2016).

Der Forschungsoutput muss dabei allerdings nicht, einseitig' in Form von Publikationen des Fachgebiets für die Unternehmungspraxis verlaufen. Es wird auch danach gestrebt, mit der Unternehmungspraxis gemeinsam zu publizieren, um so die Sichtbarkeit der Forschungsaktivitäten in der Praxis zu erhöhen. Illustrativ sei in diesem Zusammenhang auf die gemeinsam mit dem Geschäftsführer von Insiders Technologies, Werner Weiss, generierte Praxishandreichung ,Enjoy digital!' (vgl. Weiss/Müller-Seitz, 2019) verwiesen. Ähnliches gilt für die mit dem Kollegen Seiter (Universität Ulm) und dem für Smart-City-Initiativen im deutschsprachigen Raum zuständigen Leiter von IBM, Patrick Wenz, angefertigte Kurzmonographie zu Smart Cities (vgl. Müller-Seitz/Seiter/Wenz, 2016). Nebst der reinen Sichtbarkeit bringen Transferpublikationen sowie kooperativ erstellter Output den Vorteil mit sich, dass sich dadurch die Legitimation der Forschungsaktivitäten des Fachgebiets in der Unternehmungspraxis erhöht.

Auch die Vermittlung von Masterarbeiten und die Betreuung externer Promotionen lassen sich hier einordnen. Dies führt üblicherweise zu einer Intensivierung des Austauschs mit der Unternehmungspraxis und damit einhergehenden besseren Möglichkeiten, Datenzugänge für empirische Studien für wissenschaftliche Fachzeitschriftenbeiträge zu erhalten.

Es soll allerdings nicht verschwiegen werden, dass die Betreuung derartiger Praxiskooperationen auch durchaus sehr viel Zeit und Geduld in Anspruch nehmen kann. Zu nennen wäre hier etwa die Hürde des rechtlichen Interessensabgleichs zwischen der TUK und den Praxispartnern in Form von Vertraulichkeitsvereinbarungen. Die möglicherweise divergierenden Interessen des Fachgebiets an Publikationen und damit einhergehender Transparenz und Sicherstellung der Qualität einer Masterarbeit oder Promotion stellen aus Sicht der Praxispartner vielfach schmerzhafte Einschnitte dar. Umgekehrt möchte auch die TUK nicht auf etwaige Rechte an Forschungsergebnissen verzichten oder das Fachgebiet auf Publikationsmöglichkeiten. Letztlich handelt es sich also hierbei um ein zweischneidiges Schwert, wobei es gilt, die Interessen beider Parteien sorgfältig auszutarieren. Erfahrungsgemäß sind die Kooperationen auch nicht kurzfristig anzubahnen, son- dern bedürfen des Aufbaus von Vertrauen und langfristiger Pflege, um wechselseitig als attraktiv wahrgenommen zu werden.

\subsection{Schnittstelle Lehre/Praxis}

Die Schnittstelle von Lehre und Praxis wird durch die konsequente Einbindung der Unternehmenspraxis in die verschiedenen Veranstaltungen gewährleistet. Diesbezüglich kommen die folgenden Formate zum Einsatz:

- Problemorientiertes Lernen erfolgt unter Anleitung in Kleingruppen. Bei diesem Lehrformat werden die Studierenden mit aktuellen Beispielen aus der Forschungs- und Unternehmenspraxis konfrontiert und entwickeln eigenständig Lösungsansätze für die vorgestellten Problemlagen.

- Als Beispiel kann hier die Kooperation mit dem mittelständischen Software-Anbieter Insiders Technologies im Rahmen der Masterveranstaltung Innovation Management betrachtet werden. Im Anschluss an die Auseinandersetzung mit den Grundlagen des Innovationsmanagements und einem Fokus auf das Themenfeld Kreativität begleiteten die Studierenden einen Tag lang ein Software-Entwicklungsteam von Insiders Technologies, um gruppenbasierte Kreativität zu untersuchen. Kurze Zeit später stand ein Besuch im Atelier von Dietmar Brixy an, einem renommierten abstrakten Expressionisten, um so ein Verständnis für individuelle Kreativität zu erlangen. Mit Blick auf die Abschlusspräsentationen mussten die Studierenden sodann eine Konzeption für gruppenbasierte sowie individuelle Kreativität ausarbeiten und aufzeigen, wie einerseits Software-Entwicklungsteams von Brixy lernen können und andererseits wie der Künstler Brixy von dem Team bei Insiders Technologies lernen kann.

- Eine solche Lehrform ist primär für das Masterstudium vorgesehen. Zielsetzung dabei ist es, dass die Studierenden Forschungslücken in dem behandelten Themenbereich identifizieren, eine Forschungsleitfrage für eigene Projekte entwickeln und dann eine Studienarbeit erstellen. Dazu werden zunächst vorwiegend qualitative, theoriebildende, punktuell aber auch quantitative, theorietestende sozialwissenschaftliche Forschungsmethoden vorgestellt und im Kurs kritisch reflektiert.

- Im Rahmen der digitalen Transformation gängig vorzufindende Managementinstrumente, wie etwa Lego Serious Play oder Design Thinking, kommen ebenfalls punktuell je nach Thematik zum Einsatz.

- So wurde Lego Serious Play im Rahmen verschiedener Vorlesungen genutzt, um Studierende spielerisch für Fragestellungen der Digitalisierung zu sensibilisieren. In der Veranstaltung ,Innovation Management' kommt eine Einheit zum Design Thinking zum Einsatz, um die Studieren- 
den so an agile Managementinstrumente heranführen zu können.

\section{- Gastvorträge, Exkursionen und der Top Students Club} runden die Schnittstelle von Lehre und Praxis ab. Dabei wird auf ein etabliertes und sich ständig erweiterndes Netzwerk von Vertretern aus der Unternehmenspraxis zurückgegriffen.

- Im Wintersemester hat sich beispielsweise eine Kooperation mit der Deutschen Telekom etabliert, bei der im Rahmen der Abschlusspräsentation eine Exkursion zur Deutschen Telekom nach Bonn und Darmstadt integriert wird. In Bonn konnten die Studierenden so beispielsweise Einblicke in die T-Gallery erhalten, in der IT-basierte Zukunftsvisionen aus Sicht der Deutschen Telekom präsentiert werden.

- In den so genannten Top Students Club werden die besten $10 \%$ der Studierenden des Fachgebiets eines jeden Semesters aufgenommen und erhalten Informationen in Form eines Newsletters über die Geschehnisse am Fachgebiet sowie exklusive Einladungen und Zugang zu Praxispartnern. In Forms von Workshops, Fallstudienwettbewerben oder Kaminabenden wird Studierenden die Möglichkeit geboten, sich mit hochrangigen Vertreterinnen und Vertretern $\mathrm{zu}$ Digitalisierungsthemen im kleinen Kreis auszutauschen, was bisher u. a. mit der BASF, der Deutschen Telekom oder Porsche Consulting erfolgte.

\section{Fazit}

Zielsetzung des Beitrags war es, eine Innenansicht in Form eines Werkstattberichts zu liefern, wie anhand des Fallbeispiels des Fachgebiets für Strategie, Innovation und Kooperation an der TUK das Management der digitalen Transformation Eingang in Forschung, Lehre und Praxisaktivitäten findet. Der Experimentierfreude und Bereitschaft, neue Impulse mit aufzunehmen, sind jedoch weiterhin keine Grenzen gesetzt und es wird fortwährend nach neuen Impulsen für die drei Aufgabenfelder gesucht, um auch in $\mathrm{Zu}$ kunft den State-of-the-Art abbilden und selbst mitgestalten zu können.

Abschließend sei jedoch darauf hingewiesen, dass die von Dyson im Eingangszitat angemahnte Plackerei durchaus auch Freude bereitet, indem sie dem menschlichen Lerntrieb entspricht. Der Beitrag will daher zum eigenen Experimen- tieren anregen und hofft, auf Basis der geschilderten Werkstatt-Beispiele konstruktive Impulse liefern zu können.

\section{Literatur}

Corsten, H., Gössinger, R., Müller-Seitz, G., Schneider, H. (2016): Grundlagen des Technologie- und Innovationsmanagement. 2. Aufl. Vahlen. Danner-Schröder, A., Müller-Seitz, G. (2017): Qualitative Methoden in der Organisations- und Managementforschung - Ein anwendungsorientierter Leitfaden für Datensammlung und -analyse. Vahlen.

Danner-Schröder, A., Müller-Seitz, G. (im Druck): Temporal Co-Dependence between Temporary and Permanent Organizing: Tackling Grand Challenges in the Case of the Refugee Crisis in Germany, in: Research in the Sociology of Organizations.

Dobusch, L., Dobusch, L., Müller-Seitz, G. (2019): Closing for the Benefit of Openness? The Case of Wikimedia's Open Strategy Process, in: Organization Studies, 40(3), 343-370.

Hilgers, D., Müller-Seitz, G., Piller, F. (2010): Benkler Revisited - Venturing Beyond the Open Source Software Arena?, in: ICIS 2010 Proceedings. Paper 97.

Kaiser, S., Müller-Seitz, G., Lopes, M. P., Cunha, M. P. e. (2007): WeblogTechnology as a Trigger to Elicit Passion for Knowledge, in: Organization, 14(3): 391-412.

Kor, G. (2019): Winning in the Digital Economy - The Hard Slog Way. Elektronisch veröffentlicht unter der URL: https://www.linkedin.com/pul se/winning-digital-economy-hard-slog-way-geraldine-kor. Letzter Abruf: 17.10.2019.

Lange, K., Sydow, J., Müller-Seitz, G., Windeler, A. (2013): Financing Innovations in Uncertain Networks - Roadmap Gap Filling in the Semiconductor Industry, in: Research Policy, 42(39): 647-661.

Lingnau, V., Müller-Seitz, G., Roth, S. (Hrsg. 2018): Management der digitalen Transformation: Interdisziplinäre theoretische Perspektiven und praktische Ansätze. Vahlen.

Müller-Seitz, T., Beham, F., Thielen, T. (2016): Die digitale Transformation der Wertschöpfung, in: Controlling \& Management Review (6): 24-31.

Müller-Seitz, G., Braun, T. (2013): Wissenschaftliche Abschlussarbeiten in der Betriebswirtschaftslehre erfolgreich abfassen, Pearson.

Müller-Seitz, G., Seiter, M., Wenz, P. (2016): Was ist eine Smart City?, SpringerGabler

Müller-Seitz, G., Weiss, W. (2019): Strategien zum Umgang mit der digitalen Transformation...aus der Sicht eines mittelständischen „Hidden Champions". Vahlen.

Müller-Seitz, G., Zühlke, D., Braun, T., Gorecky, D., Thielen, T. (2018): Netzwerkbasierte Geschäftsmodellinnovationen - Das Beispiel der Industrie 4.0-Anlage SmartFactoryKL. Die Unternehmung, 72(2), 146-168.

Sydow, J., Schüßler, E., Müller-Seitz, G. (2016): Managing Interorganizational Relations - Debates and Cases, Palgrave/Macmillan Publishers.

Technische Universität Kaiserslautern (2019): Hochschulentwicklungsplan 2025. Kaiserslautern: Technische Universität Kaiserslautern.

Thielen, T. (2019): Netzwerkbasierte Geschäftsmodellinnovationen. Untersuchung des Innovationsprozesses von Geschäftsmodellen auf Whole Network Level vor dem Hintergrund von Industrie 4.0 Shaker.

Weiss, W., Müller-Seitz, G. (2019): Enjoy digital! Erfolgsrezepte für das Management der digitalen Transformation. Vahlen. 